\title{
First Metatarsophalangeal Arthrodesis Malunion Revision to Silicone Implant Arthroplasty
}

\author{
Paul J Carroll* and Jonathan J Sharpe \\ Division of Podiatric Surgery, Orthopedic Associates of Lake County, USA
}

*Corresponding author: Paul Carroll, Fellow, Northern Ohio Foot and Ankle Reconstruction Fellowship, Division of Podiatric Surgery, Orthopedic Associates of Lake County, Lake Health, 7551 Fredle Dr. Concord, OH-44077, USA, Tel: 440-3509595, Fax: (440)-357-1905

\begin{abstract}
A shortened first metatarsophalangeal joint (MTPJ) fusion can be a debilitating complication of arthrodesis surgery. The most commonly accepted treatment is revision of the first MTPJ revisional arthrodesis with lengthening utilizing bone graft. To our knowledge, there is no published literature on first MTPJ short malunion correction using monorail distraction and arthroplasty with a silastic implant. A case is presented of a young active 32-year-old individual who presented to our office following a first MPJ arthrodesis and first tarsometatarsal joint (TMTJ) arthrodesis with severe shortening of the metatarsal. The patient underwent a two-stage procedure consisting of a fusion take down with external monorail distraction fixator, followed by first MTPJ arthroplasty with a silastic implant.
\end{abstract}

\section{Keywords}

Distraction arthroplasty, Hallux limitus, Transfer lesions, Metatarsalgia, Malunion

\section{Introduction}

First metatarsophangeal joint (MTPJ) arthritis has been reported in approximately 2.5 to $7.8 \%$ of people in the US [1,2]. If conservative treatment fails, surgeons have the option of performing either a joint salvage procedure, implant arthroplasty, or fusion for treatment of the painful arthritis. First MTPJ fusion is one of the most common surgical treatment options for advanced arthritis of the first MTPJ. If a first MTPJ fusion is performed, complications may arise if excess bone is resected causing a shortened malunion. A shortened first MTPJ fusion can be a debilitating complication of an arthrodesis. It often results in transfer pain to the lesser metatarsals, which can cause stress fractures and metatarsalgia. There is a lack of proper hallux purchase and propulsion typically lateral transfer of weightbearing stress. The mechanical imbalance may also cause digital contractures as well. The current recommended treatment of this complication is re-establishing length of the first MTPJ through bone grafting [3]. It is most commonly performed with a bone graft; however, callus distraction has been described in the literature as well. The purpose of this paper is to present a case of patient who underwent a two-staged procedure for a shortened malunion after a first MTPJ and first tarsometatarsal joint (TMTJ) fusion on the ipsilateral foot. The procedure included re-establishing length as well as motion of the joint with a silicone implant. To our knowledge this has not been reported in the literature.

\section{Case Presentation}

A 32-year-old male was seen by the senior author (JJS) for a burning, tingling and chronic pain in his left foot. The patient is an active member of the military as well as a prior CrossFit enthusiast. Prior to consultation, this patient had a first TMTJ and first MTPJ arthrodesis performed on the ipsilateral left foot at an outside institution. However, this procedure did not provide him with relief and he reported worsening symptoms postoperatively. Becoming increasingly frustrated, because the pain is restricting his duties, he was seen in our office (JJS) for a second opinion (Figure 1). On clinical observation, his first ray was severely shortened and rigid with loss of hallux purchase on weightbearing. Clinically, he had a solid arthrodesis of the first ray at the MTPJ and TMTJ. Calluses were noted plantar to 

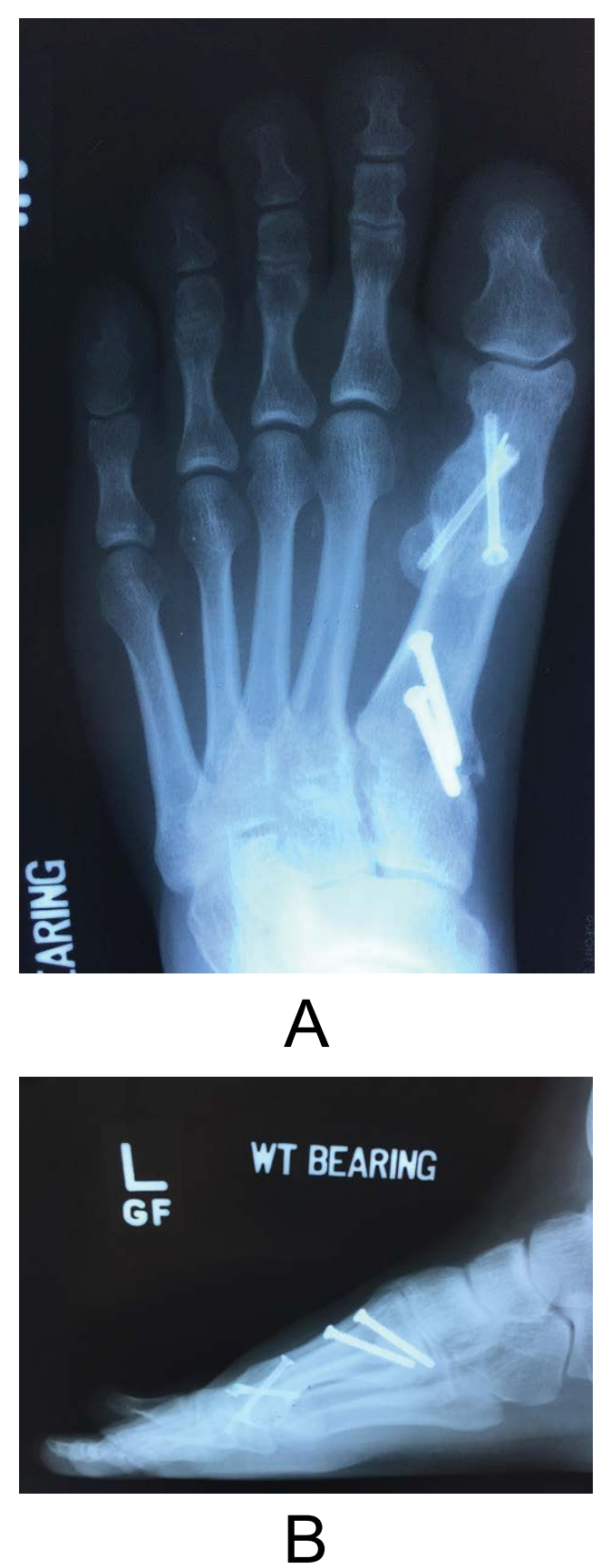

Figure 1: First MTPJ and TMTJ shortened malunion with screw fixation of both MTPJ and metatarsal-cuneiform joint fusion. Note the severe hallux elevation off of the weightbearing surface on the lateral projection as well.

metatarsal heads two through five on the left foot. After a physical and gait exam, as well as reviewing his radiographs it was determined the cause of his pain was from the shortening and malunion of his first ray on the left foot. Upon initial evaluation, it was noted that there was diminished vibratory sensation of nerve distribution of L-5, S-1. A neurology consultation was obtained; however, it was noted he did not have any neurologic pathology. This narrowed the focus of his pain to the pathomechanics of his foot. The motion of his first interphalangeal joint (IPJ) was within normal range of motion without crepitus or pain. However, his IPJ range of motion was not adequate enough to compensate for both the first TMTJ fusion and shortened first MTPJ fu- sion, as the hallux did not purchase the ground when weightbearing. The sequential arthrodesis of the first TMTJ and first MTPJ complicated the revisional options.

Extensive surgical consultation was performed on multiple visits. The patient adamantly refused any revisional arthrodesis secondary to his prior experience, despite ample discussion and education that this would be his best revisional option. Ultimately, it was decided a two-stage procedure first re-establishing first ray length followed by re-establishment of a first MPTJ with a silicone implant could be a surgical option with extensive discussion on the lack of literature or experience to guide this decision. The pathomechanics behind his pain by the first TMTJ and the shortened first MTPJ fusion were discussed with the patient. We discussed the complexity of the revision as well with subsequent arthrodesis to the first TMTJ and MTPJ and how the lack of motion in these two joints affects the mechanics of the first ray and propulsion. To accommodate the new joint the soft tissue surrounding the joint would need to be elongated with which require the use of an external monorail fixator after the malunion takedown. Once proper length was achieved, a second procedure was to be performed to introduce the silastic implant. After this thorough discussion with the patient and his family the patient agreed to undergo the staged procedure.

The patient was placed supine on the operating room table with an ipsilateral hip bump. A pneumatic calf tourniquet was applied for hemostasis. After sterile preparation and exsanguination, approximately an eight-centimeter linear incision was made or the dorsal aspect of the first TMTJ crossing the first MTPJ. Full thickness skin, subcutaneous and periosteal flaps were made exposing the first MTPJ malunion. All previous deep hardware was removed. An osteotomy was made through the first MTPJ malunion perpendicular to the weightbearing surface (Figure 2). Four $3.0 \mathrm{~mm}$ partially threaded half pins were placed along the longitudinal axis of the first ray. Two were placed in the proximal phalanx and two in the metatarsal and a SIDEKICK ${ }^{\mathrm{m} m}$ CORETRAK ${ }^{\mathrm{m} m}$ monorail fixator (Wright Medical, Memphis, TN) was applied. An osteotomy was performed through the malunion apex perpendicular to the weightbearing surface and the hallux was distracted approximately one center on the OR table. The incision was irrigated, and a layered closure was performed. The patient remained non-weight bearing following the procedure. Over the next month, the patient distracted the osteotomy by 0.25 millimeters $(\mathrm{mm})$ daily start post-operative day one. The distraction regiment closely follows distraction osteogenesis, however, the goal was to establish a gap greater than one centimeter between the proximal phalanx and the metatarsal to allow for fitting of the silicone implant and to elongate the hallux to the length of the second digit. The contralateral foot was used as a guide for distraction length as well. At a distraction rate of $0.25 \mathrm{~mm}$ daily, this allowed for safe soft tissue elongation and avoid neurovascular compromise. 


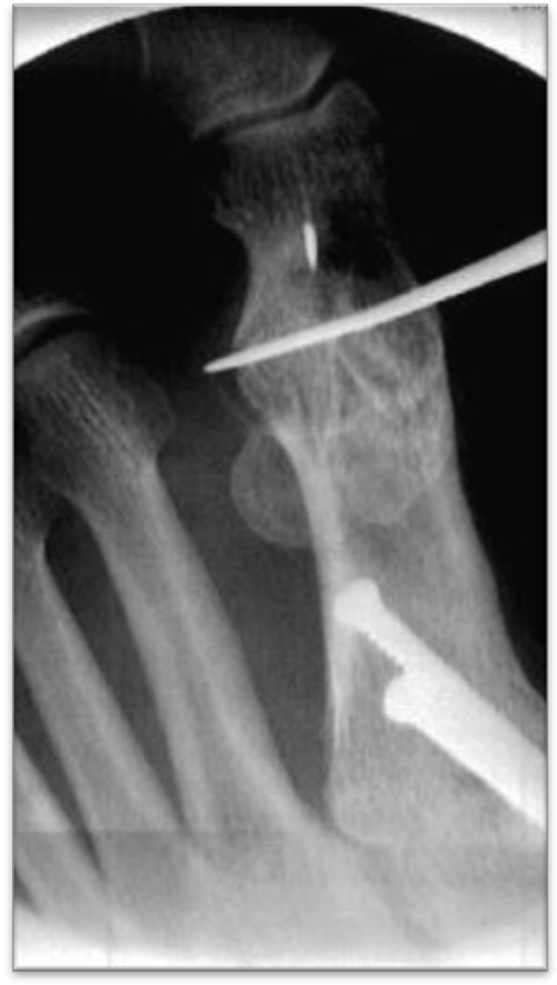

A

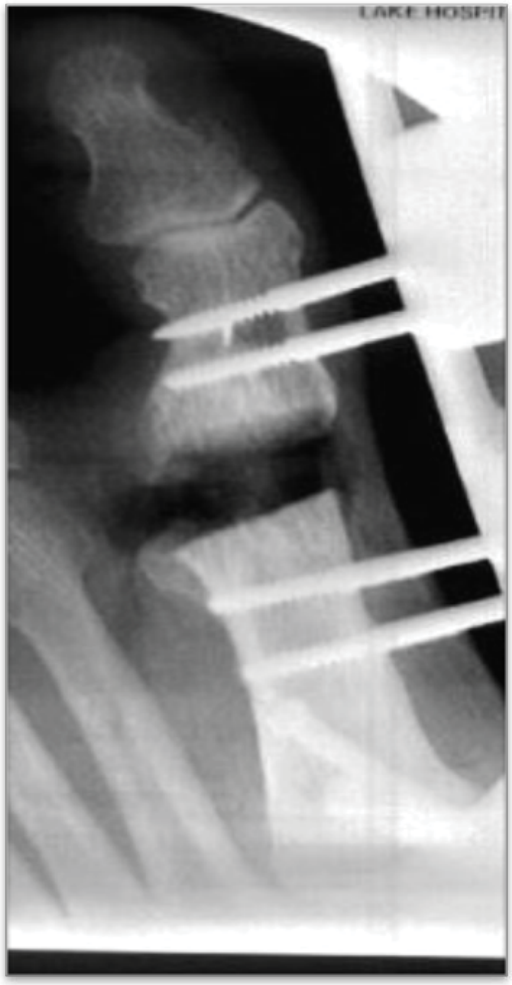

B

Figure 2: Intraoperative osteotomy through the previous first MPTJ malunion after deep hardware removal. Application of SIDEKICK ${ }^{\mathrm{TM}}$ CORETRAK $^{\mathrm{TM}}$ monorail fixator (Wright Medical, Memphis, TN) for distraction of the osteotomy.

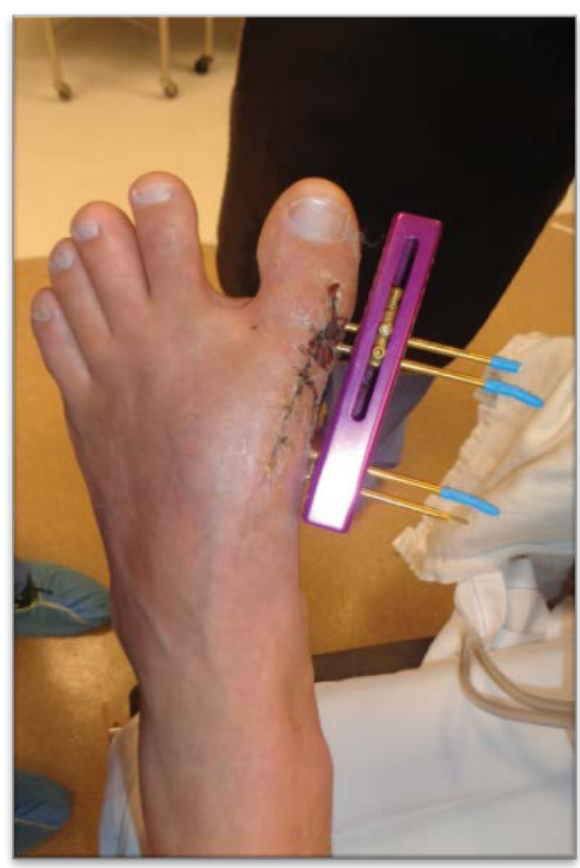

A

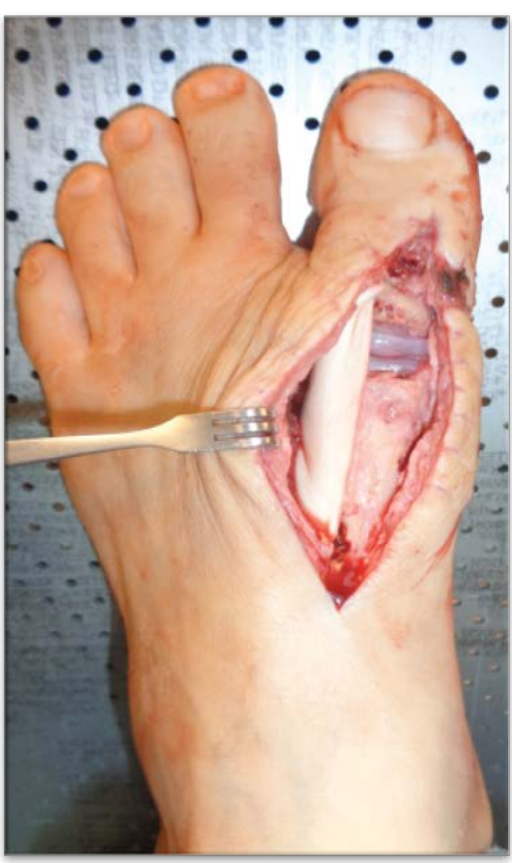

B

Figure 3: Joint distraction clinically prior to second stage implant placement. Swanson flexible Hinge Toe implant with graftjacket $^{\circledR}$ (Wright Medical, Memphis, TN) reflected medially and EHL retracted laterally displaying the silicone implant placement.

At time of the second operation it was noted there was superficial skin sloughing at the distal pin sites, which did not extend below the dermis. Additionally, there were no clinical signs of infection at the incision site (Figure 3A).
The patient returned to the operating room one month following the initial procedure for the implantation of silastic implant. The patient was positioned in the same manner as the previous surgery. The external monorail fixator was removed. After sterile preparation, utilizing the prior incision, a full thickness incision was made. Full 
thickness flaps were created. The fibrocartilage and scar tissue were debrided from the distraction void. A Swanson flexible hinge toe silicone ${ }^{\circledR}$ implant (Wright Medical, Memphis, TN) was implanted in the recreated first MTPJ. The joint was irrigated, a thin Graftjacket ${ }^{\circledR}$ (Wright Medical, Memphis, TN) allograft was wrapped around the implant and phalangeal base and first metatarsal head to create joint capsule and for structural containment. The extensor tendons were not included in the graft wrap (Figure 3B). The incision was irrigated a second time and a layered closure was performed. During the recovery period underwent aggressive physical therapy. He was fitted for orthotics three months after surgery and has reported an improvement in his level of pain. Fifteen months following the procedure the patient's pain has improved. He is currently in orthotics, back to marching and light exercise in the military with a tolerable level of discomfort. He reports a better gait pattern, which was seen clinically as well. The patient reported being satisfied with his surgical result at 15 months (Figure 4).

\section{Discussion}

We present a unique case of a shortened first MTPJ malunion corrected with a distraction arthroplasty followed by a silicone implant. Currently, the most commonly accepted treatment for correction a shortened first MTPJ malunion is bone block arthrodesis. Many authors have published positive results of bone block arthrodesis of a first metatarsophalangeal joint in setting of a failed arthroplasty. Myerson, et al. [3] reported an increase of 40 points in pre and post-operative AOFAS scores (39 to 79) following restoration of a MTPJ arthrodesis using a bone graft. He also reported a $79.1 \%$ fusion in 24 patients undergoing distraction arthrodesis of the first MTPJ [3]. Additionally, on average 13 millimeters of length of was restored with bone grafting.
Our decision to deviate from the more commonly accepted treatment was influenced by several factors. The first, was the additional fusion of the first TMTJ joint. By locking up the first ray, this significantly increasing stress in adjacent joints, in this case the IPJ of the hallux. In an isolated first MPTJ fusion, the IPJ can accommodate the increased stress. However, in this case, fusion of two consecutive joints in the first ray may have had deleterious effects on the foot mechanics. We believed re-establishing motion of the first MTPJ would decrease stress of the IPJ resulting in pain reduction. Another contributing factor was the severe shortened malunion of the first MTPJ fusion. Most notably, the patient adamantly refused a revisional arthrodesis after extensive discussion.

The Swanson flexible hinge toe silicone ${ }^{\circledR}$ (Wright Medical, Memphis, TN) implant was used for its distinct advantage compared to newer metal implants. The Swanson ${ }^{\circledR}$ implant contains a silicone spacer, increasing the longitudinal length of the implant. This was advantageous in this case because additional length needed to correct the severe first ray shortening. Interpositional arthroplasty would not have corrected the severe length deficit and likely would not have re-established hallux purchase on the ground either. This could not be accomplished with a bi-polar implant in our opinion either after significant lengthening and distraction. A hemi-implant was not an option secondary to prior arthrodesis, no joint surface or cartilage was left on either side of the first MPTJ to accommodate a hemi-implant and again, not enough volume to maintain the distraction to proper length and hallux purchase. Silicone implants were introduced in 1967 by Swanson, to create a joint spacer following excision of the base of the proximal phalanx $[4,5]$. This made the implant ideal for this particular case. Some surgeons advocate against their use because

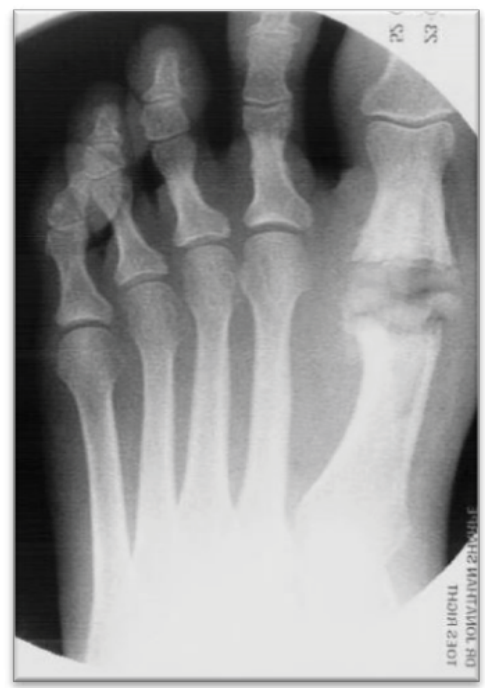

A

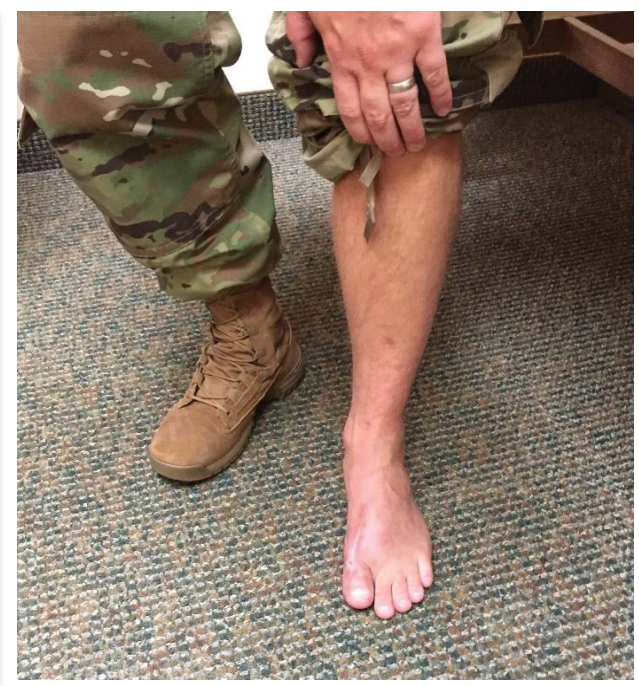

B

Figure 4: Intra-operative radiographic photo. Compare to the pre-operative image where the hallux IPJ lies adjacent to the midshaft of proximal phalanx of second toe pre-operatively vs. at the level of the PIPJ post-operatively. Postoperative clinical images at final follow up. 
detritic synovitis, silicone synovitis, fatigue, lymphatic migration, erosive osteolysis and silicone lymphadenopathy can occur [6]. In a retrospective study of silastic implants by Bonnet, et al. he found $63 \%$ of patients were completely satisfied eight years after surgery [7]. He also reported $78 \%$ patients had pain relief initialing following the procedure. In a similar study by Samer, et al. she reported a $78 \%$ pain relief in 83 people 8.5 years post-operatively [8].

The decision to use a monorail external fixator allowed for gradual soft tissue elongation and re-establishment of proper first ray length in preparation of the implant. Our intention of starting distraction at post-operative day one, rather than waiting until day seven, was to prevent osteogenesis. The hallux was lengthened to match the contralateral foot and adjacent second digit.

One minor complication that did occur was the patient developed superficial skin sloughing at the distal pin site during the distraction period of the external fixator. This did not extend deeper to the dermis. We believe the skin sloughing was caused by two factors. The first was potentially the close proximity of the proximal phalanx pins to each other. This could have resulted in a too small of a skin bridge between the pins. Secondly, we started distraction at post-operative day one, the absence of a latent period may not have allowed the skin to recover from the surgery.

There are limitations to our singular case study. First, this a retrospective case study. There is also relatively short term follow up at only 15 months. Silicone implants may have a limited survivorship and with this patient's young age and he may require additional revisional surgeries.

\section{Conclusion}

This is a case of a successful distraction arthroplasty of a shortened first MTPJ and first TMTJ fusion. The patient was able to return to his military duties and avoid discharge. However, we recommend more term follow up as well as prospective studies to determine if this procedure can be a long-term solution to this surgical complication.

\section{Conflict of Interest}

None.

\section{Financial Disclosure}

Dr. Sharpe is a paid consultant of Wright Medical.

Paul Carroll (Principle Author): 70\%, Collected data, interpreted data, wrote manuscript acted as corresponding author.

Jonathan Sharpe (Co-author): $30 \%$ Senior author, supervised development of work.

\section{References}

1. Ho B, Baumhauer J (2017) Hallux rigidus EOR 2: 13-20.

2. Munteanu S, Landorf K, McClelland J, Roddy E, Cicuttini F, et al. (2017) Shoe-stiffening inserts for first metatarsophalangeal joint osteoarthritis (the SIMPLE trial): Study protocol for a randomized controlled trial. Trials 18: 198.

3. Myerson M, Schon L, McGuigan F, Onzur A (2000) Result of arthrodesis of the hallux metatarsophalangeal joint using bone graft for restoration of length. Foot Ankle Int 21: 297306.

4. Swanson A, Biddulph S, Hagert C (1971) A silicone rubber implant to supplement the Keller toe arthroplasty. NY Univ Inter Clin Inform Bull 10: 7-14

5. Swanson A, Lumsden R, Swanson G (1979) Silicone implant arthroplasty of the great toe: A review of single stem and flexible hinge implant. Clin Orthop Relat Res 142: 30-42.

6. Needleman L (1995) The microscopic interaction between silicone and the surrounding tissues. Clin Podiatr Med Surg 12: $415-423$.

7. Bonet J, Taylor D, Lam A, Eveleigh W, Keane L (1998) Retrospective analysis of silastic implant arthroplasty of the first metatarsophalangeal joint. J Foot Ankle Surg 37: 128-134.

8. Samer M, Ng A, Clough T (2012) The long-term outcome of silastic implant arthroplasty of the first metatarsophangeal joint: A retrospective analysis of one hundred and eight feet. Int Orthop 36: 1865-1869. 